\title{
EFFICIENT LINEAR SCHEMES WITH UNCONDITIONALLY ENERGY STABILITY FOR THE PHASE FIELD ELASTIC BENDING ENERGY MODEL
}

\author{
XIAOFENG YANG * AND LILI JU ${ }^{\dagger}$
}

\begin{abstract}
In this paper, we study efficient numerical schemes of the classical phase field elastic bending energy model that has been widely used to describe the shape deformation of biological lipid vesicles, in which the free energy of the system consists of an elastic bending energy, a surface area constraint and a volume constraint. One major challenge in solving such model numerically is how to develop appropriate temporal discretizations in order to preserve energy stability with large time step sizes at the semi-discrete level. In this paper, we develop a first and a second order time stepping schemes for this highly nonlinear and stiff PDE system based on the "Invariant Energy Quadratization" method. In particular, the resulted semi-discretizations lead to linear systems in space with symmetric positive definite operators at each time step, thus can be efficiently solved. In addition, the proposed schemes are rigorously proved to be unconditionally energy stable. Various numerical experiments in $2 \mathrm{D}$ and $3 \mathrm{D}$ are presented to demonstrate the stability and accuracy of the proposed schemes.
\end{abstract}

\section{INTRODUCTION}

Lipid vesicles (liposomes) are closed structures in which (at least) one lipid bilayer separates an aqueous inner compartment from the bulk external aqueous medium, as in membranes of contemporary biological cells. Lipid vesicles are ubiquitous in biological systems and work as the building block to cellular life. Over $70 \%$ of cell membranes are composed of lipids with a bilayer structure. There have been many experimental and analytic studies on the configurations and deformations of elastic vesicle membranes [1-3,10,13,15, 27, 28, 32, 37, 41]. In the pioneering work of Canham, Evans and Helfrich $[5,6,17,23]$, a continuum bending elasticity model was developed for the single-phase membrane, where the dynamical deformation of the shapes of a lipid membrane, is determined by the so-called sharp interface elastic bending energy. In the last decade, the diffuse interface approach, or called as the phase field method, had been first applied in the field $[10,13,15]$ to approximate the elastic bending energy for the lipid vesicles, in which, the mean curvature of the membrane surface is replaced by a modified elastic bending energy functional based on a phase field variable. The evolution equations are then resulted from the variation of the action functional of the free energy.

Key words and phrases. Phase-field, Allen-Cahn, Linear, Elastic Bending Energy, Invariant Energy Quadratization, Energy Stability.

* Corresponding author, Department of Mathematics, University of South Carolina, Columbia, SC 29208, USA and Beijing Center for Scientific and Engineering Computing, Beijing University of Technology, Beijing 100124, China. Email: xfyang@math.sc.edu.

$\dagger$ Department of Mathematics, University of South Carolina, Columbia, SC 29208, USA. Email: ju@math.sc.edu. 
The idea of phase field approach can be dated back to the ancient studies a century ago [34,40]. Such method considers the free moving interface between multiple material components as a continuous, but steep change of some physical material properties, for instances, density or viscosity, thus a continuous phase field variable is introduced and the interface is represented by a thin but smooth transition layer. One major advantage of this method is that the free interface can be automatically tracked without imposing any mathematical conditions (e.g. Young-Laplace junction condition $[16,26,33]$ in sharp interface model) on the moving interface, thus it provides an easy treatment of topological changes of the interface. Furthermore, the phase-field model is usually derived from an energy-based variational formalism, thus it leads to well-posed nonlinearly coupled systems that satisfy thermodynamics-consistent energy dissipation laws. For the microstructures of the lipid vesicles, the phase field models have been applied to study the shape transformations in various situations effectively, for example, multi-component vesicles $[19,24,44]$, vesicle-substrate adhesion $[38,48]$, and vesicle-vesicle adhesion problems [20], dynamics of vesicles in fluids [10,11], etc. Meanwhile, through asymptotic analysis, the consistency between the phase field formulation and the original sharp interface problem (Willmore flow) has been established in $[12,43]$. Recently, the phase field approach has been successfully employed in many fields of science and engineering ever since and it now becomes one of the major modeling and computational tools for the study of interfacial phenomena (cf. $[4,7,9,13,25,29-31,39]$ and the references therein).

From the numerical point of view, for phase field models, it is specifically desired to design numerical schemes that could preserve the thermo-dynamically consistent dissipation law (energy-stable) at the discrete level irrespectively of the coarseness of the discretization. The preservation of such laws is critical for numerical methods to capture the correct long time dynamics of the system. Furthermore, the energy-law preserving schemes provide flexibility for dealing with the stiffness issue of the model. Although a variety of numerical algorithms have been developed for the phase field elastic bending energy (PF-EBE) model, that was proposed and studied in $[10,13,15]$, we notice that there are very few successful attempts in designing efficient energy stable schemes due to the strong stiffness issue of the interfacial width, as well as the complicated nonlinear terms induced from the elastic bending energy, the volume and the surface area constraints. In [8], the authors developed a first order in time, linear scheme based on the stabilization approach $[35,36,46]$, where the nonlinear terms are all treated explicitly and two extra first order linear stabilizers are added to improve the stability. However, the energy stability can be only obtained by pre-assuming that the analytic solutions satisfy the maximum principle, but that is unable to be proved yet. Moreover, the authors did not consider the fixed surface area constraints. In [45], the authors developed some high-order accurate linear schemes where the nonlinear terms are treated by the so-called explicit exponential time differencing Runge-Kutta (ETD-RK) approach. However, the schemes involve extra splitting errors and their energy stabilities were not able to be theoretically proved yet, even though orders of accuracy and numerical stability were illustrated by extensive numerical experiments. Overall, briefly speaking, most of the existing methods are either low-order accurate in time, or their energy stabilities can hardly be obtained in theory.

It is remarkable that, for some simple phase field models, e.g., the Cahn-Hilliard equation or the Allen-Cahn equation, there exist a few well developed techniques to discretize the nonlinear terms, (e.g. double well potentials), while preserving the energy stabilities, e.g. the convex splitting approach (implicit) [18] or the stabilization approach (explicit) $[9,35,46]$. However, 
these two methods are not applicable when dealing with the PF-EBE model due to some essential difficulties, including that (i) implicit schemes based on the convex splitting approach require iterative solution of large systems at each time step while the linear schemes based on the stabilization method often requires relatively small time step sizes; (ii) the analytical solution of the PDE system may not satisfy the maximum principle; (iii) it is questionable whether the nonlinear potential can be split into the combinations of some convex functionals; (iv) the surface area or the volume constraints still present substantial stiffness and huge barriers to obtain the energy stability of the numerical schemes.

Therefore, the main purpose of this paper is to develop some effective and efficient numerical schemes for solving the PF-EBE model in $[10,13,15]$. We adopt a novel approach, called the Invariant Energy Quadratization (IEQ) method, to linearize the diverse nonlinear functionals while preserving the energy stability. Actually such method has been successfully applied in the context of other phase field models (cf. $[21,22,47]$ ) for polynomial type potentials. But when it is applied to the PF-EBE model, there are still some new challenges due to the nonlinearities in various terms, in particular, the fixed volume and surface area constraints. The idea of IEQ method is to introduce some transformations to enforce the free energy density as an invariant quadratic (since the nonlinear potential is usually bounded from below) functionals in terms of new, auxiliary variables. Then the governing system of equations can be reformulated upon the new variables to an equivalent system. Instead of considering linear stabilizers or analyzing the convexity for those nonlinear functionals, all nonlinear terms in the new system can be treated semi-explicitly, which in turn produces a system of linear elliptic equations. Based on this new approach, we develop first and second order schemes, which (i) are accurate (up to second order in time); (ii) are stable (energy dissipation law holds); and (iii) are efficient and easy to implement (only need to solve a linear system with symmetric positive definite operator at each time step). To the best of our knowledge, the proposed schemes in this paper are the first such schemes for the PF-EBE system that are theoretically proved to have all these desired properties.

The rest of the paper is organized as follows. In Section 2, we briefly introduce the PF-EBE model and its PDE energy law. In Section 3, we develop linear numerical schemes with respective first order and second order accuracy for simulating the model, and then rigorously prove their well-posedness and unconditional energy stabilities. Various 2D and 3D numerical experiments are tested in Section 4 to validate the accuracy and efficiency of the proposed schemes. Finally, some concluding remarks are given in Section 5.

\section{The Phase Field ElAStic Bending ENERGy MODEL AND ITS ENERGY LAW}

We now give a brief introduction to the PF-EBE model. The equilibrium shape of a vesicle membrane is determined by minimizing the elastic bending energy (cf. $[5,6,17,23]$ ),

$$
E=\int_{\Gamma}\left(a_{1}+a_{2}\left(H-c_{0}\right)^{2}+a_{3} K\right) d s
$$

where $\Gamma$ is a smooth compact surface in the domain $\Omega \in \mathbf{R}^{d}, d=2,3, H=\left(k_{1}+k_{2}\right) / 2$ represents mean curvature of the membrane surface and $K=k_{1} k_{2}$ is Gaussian curvature with $k_{1}, k_{2}$ bring two principle curvatures, $a_{1}$ is the surface tension, $a_{2}$ and $a_{3}$ are bending rigidities, and $c_{0}$ represents spontaneous curvature. 
If we consider the model to be isotropic, i.e., the spontaneous curvature $c_{0}=0$ and neglect the constants $a_{1}$ and $a_{3}$ due to the Gauss-Bonnet formula, then the elastic bending energy can be written by

$$
E=\int_{\Gamma} \frac{K}{2} H^{2} d s
$$

In the framework of phase field approach, a phase variable $\phi(\boldsymbol{x})=\tanh \left(\frac{d(\boldsymbol{x})}{\sqrt{2} \epsilon}\right)$ is defined for all $\boldsymbol{x} \in \Omega$, where $d(\boldsymbol{x})$ is the signed distance between a point $\boldsymbol{x}$ and the surface $\Gamma$, positive inside and negative outside, and $\epsilon$ is a transition parameter that is taken to be very small to characterize the width of diffusive interface or transition layer. Thus $H=-\frac{1}{2} \operatorname{tr}\left(\nabla^{2} d(\boldsymbol{x})\right)$ on the surface and one can obtain the phase field bending energy as follows [42]:

$$
E_{b}(\phi)=\int_{\Omega} \frac{\epsilon}{2}(\Delta \phi-f(\phi))^{2} d \boldsymbol{x},
$$

where $F(\phi)=\frac{\left(\phi^{2}-1\right)^{2}}{4 \epsilon^{2}}$ is the Ginzburg-Landau double well potential and $f(\phi)=F^{\prime}(\phi)=\frac{\phi\left(\phi^{2}-1\right)}{\epsilon^{2}}$.

When one uses the elastic bending energy model to describe vesicles, a fixed surface area and a fixed insider volume are often required. Corresponding to the phase field formulation, for the simplicity of representation, we define the scaled volume $V_{S}(\phi)$ and surface area function $A_{S}(\phi)$ as

$$
V_{S}(\phi)=\int_{\Omega}(\phi+1) d \boldsymbol{x}, \quad A_{S}(\phi)=\epsilon \int_{\Omega}\left(\frac{1}{2}|\nabla \phi|^{2}+F(\phi)\right) d \boldsymbol{x} .
$$

It is well known that $\frac{1}{2} V_{S}(\phi)$ approaches the volume of the vesicle and $\frac{3}{2 \sqrt{2}} A_{S}(\phi)$ converges to the surface area quadratically as $\epsilon \rightarrow 0$. If one considers the constraints of fixed volume and surface area, then the more general energy functional $E(\phi)$ includes two extra terms as follows $[10,13,15]$

$$
E(\phi)=\int_{\Omega} \frac{\epsilon}{2}(\Delta \phi-f(\phi))^{2} d \boldsymbol{x}+\frac{1}{2} M_{1}\left(V_{S}(\phi)-\alpha\right)^{2}+\frac{1}{2} M_{2}\left(A_{S}(\phi)-\beta\right)^{2},
$$

where $M_{1}$ and $M_{2}$ are two positive penalty parameters, $\alpha$ and $\beta$ denote the constants related to the volume and surface area, respectively. Throughout in this paper, we set $\alpha=V_{S}\left(\phi_{0}\right)$ and $\beta=A_{S}\left(\phi_{0}\right)$ with $\phi_{0}=\phi(t=0)$.

Adopting the Allen-Cahn relaxation dynamics (the gradient flow in $L^{2}$ space), one then obtains the governing dynamical equation for $\phi(\boldsymbol{x}, t)$ in the PF-EBE model based on the variational approach in the following

$$
\begin{aligned}
\phi_{t} & =-\frac{\delta E(\phi)}{\delta \phi} \\
& =-\epsilon\left(\Delta-f^{\prime}(\phi)\right)(\Delta \phi-f(\phi))-M_{1}\left(V_{S}(\phi)-\alpha\right)-\epsilon M_{2}\left(A_{S}(\phi)-\beta\right)(-\Delta \phi+f(\phi)),
\end{aligned}
$$

where $f^{\prime}(\phi)=\frac{3 \phi^{2}-1}{\epsilon^{2}}$. Without loss of generality, we adopt the periodic boundary condition to remove all complexities associated with the boundary integrals in this study. We remark that the boundary conditions can also be the no-flux type as $\left.\partial_{\mathbf{n}} \phi\right|_{\partial \Omega}=\left.\partial_{\mathbf{n}} \Delta \phi\right|_{\partial \Omega}=0$ where $\mathbf{n}$ is the outward normal of the computational domain $\Omega$. All numerical analysis in this paper can be carried out to the no-flux boundary conditions without any further difficulties.

Denote by $(g(\boldsymbol{x}), h(\boldsymbol{x}))=\int_{\Omega} g(\boldsymbol{x}) h(\boldsymbol{x}) d \boldsymbol{x}$ the $L^{2}$ inner product of any two functions $g(\boldsymbol{x})$ and $h(\boldsymbol{x})$, and by $\|g\|=\sqrt{(g, g)}$ the $L^{2}$ norm of the function $g(\boldsymbol{x})$. By taking the inner product of 
(2.6) with $\phi_{t}$, we obtain the energy dissipation law as

$$
\frac{d}{d t} E(\phi)=-\left\|\phi_{t}\right\|^{2} \leq 0
$$

\section{LiNEAR NUMERICAL SCHEMES WITH UNCONDITIONALLY ENERGY STABILITY}

We now construct unconditionally energy stable, linear numerical schemes for solving the PF-EBE model (2.6). The main challenging issue to solve such model numerically is about the time marching problem, i.e., how to develop suitable temporal discretizations for the nonlinear terms in order to preserve the energy stability at the discrete level.

For some simple phase field models, e.g., the classical Cahn-Hilliard equation or the AllenCahn equation, where the only nonlinear potential in the free energy is the Ginzburg-Landau double well potential (fourth order polynomial $F(\phi)$ ), there are mainly two commonly used techniques to discretize the nonlinear term (third order polynomial $f(\phi)$ ) in the PDE system while preserving the unconditional energy stability. The first one is the so-called convex splitting approach [18], where the convex part of the potential is treated implicitly and the concave part is treated explicitly. The convex splitting approach is energy stable, however, it produces a nonlinear scheme at most cases, thus the implementation is complicated and the computational cost is high. The second one is the so-called stabilization approach $[9,35,46]$, where the nonlinear term is simply treated explicitly. In order to preserve the energy stability, a linear stabilizing term has to be added, and the magnitude of that term usually depends on the upper bound of the second order derivative of the double well potential. The stabilization approach introduces a purely linear scheme, thus it is absolutely easy to implement. One immediately notices that such derivative usually does not have finite upper bound. A feasible remedy is to make some reasonable revisions to the nonlinear potential in order to obtain a finite bound, for example, a quadratic cut-off function for the double well potential. Such method is particularly reliable for those models with the maximum principle. If the maximum principle does not hold, the revisions to the nonlinear potentials may lead to spurious solutions. Moreover, one of the drawbacks of the stabilization approach is its second order version only preserves the conditional energy stability (cf. the detailed proof in [35]), i.e., the time step size is controlled by the interfacial thickness.

In addition, in $[21,22,47]$, a linearization approach was developed to discretize the polynomial type potential linearly. While this method is inspiring, it seemed that the applications are limited to the cubic polynomial term, i.e., the derivative of the fourth order polynomial type double well potential.

It is remarkable that the ideas of the convex splitting or the stabilization approach are not applicable for the PF-EBE model. The stabilization approach can not provide ideal schemes, since (i) it is uncertain whether the PDE solution could satisfy any maximum principle; (ii) we can not obtain the energy stability when applying the stabilization approach even for the first order scheme; and (iii) we expect to develop temporal second order schemes with unconditional energy stability, but the stabilization approach fails for even simpler models. Meanwhile, it is particularly not clear and questionable whether the nonlinear potentials, particularly the volume and surface area constraints, could be split into the combinations of the convex and concave parts, which excludes the convex splitting approach.

We aim to develop some more effective and efficient numerical schemes in this paper. More precisely, we expect that the schemes are efficient (linear system), stable (unconditionally energy stable), and accurate (ready for second order or even higher order in time). To this end, we 
adopt the Invariant Energy Quadratization (IEQ) approach to design desired numerical schemes, without worrying about whether the continuous/discrete maximum principle holds or a convexity/concavity splitting exists.

3.1. Transformed system. Let us define three auxiliary variables as follows:

$$
\begin{aligned}
U & =\Delta \phi-f(\phi), \\
V & =V_{S}(\phi)-\alpha, \\
W & =A_{S}(\phi)-\beta .
\end{aligned}
$$

In turn, the total free energy $(2.5)$ can be rewritten as

$$
\mathbb{E}(U, V, W)=\int_{\Omega} \frac{\epsilon}{2} U^{2} d \boldsymbol{x}+\frac{M_{1}}{2} V^{2}+\frac{M_{2}}{2} W^{2} .
$$

Then we obtain a new, but equivalent PDE system by taking the time derivative for the new variables:

$$
\begin{aligned}
\phi_{t} & =-\epsilon\left(\Delta-f^{\prime}(\phi)\right) U-M_{1} V-\epsilon M_{2} W(-\Delta \phi+f(\phi)), \\
U_{t} & =\left(\Delta-f^{\prime}(\phi)\right) \phi_{t}, \\
V_{t} & =\int_{\Omega} \phi_{t} d \boldsymbol{x} \\
W_{t} & =\int_{\Omega} \epsilon(-\Delta \phi+f(\phi)) \phi_{t} d \boldsymbol{x} .
\end{aligned}
$$

The transformed system (3.5)-(3.8) in the new variables form a closed PDE system with the following initial conditions,

$$
\left\{\begin{array}{l}
\phi(t=0)=\phi_{0}, \\
U(t=0)=\Delta \phi_{0}-f\left(\phi_{0}\right), \\
V(t=0)=V_{S}\left(\phi_{0}\right)-\alpha, \\
W(t=0)=A_{S}\left(\phi_{0}\right)-\beta .
\end{array}\right.
$$

Note that the boundary conditions of the new system are still exactly same as the the original system since the equations (3.6)-(3.8) for the new variables $U, V, W$ are only ordinary differential equations for time.

It is clear that the new transformed system still retains a similar energy dissipative law. Taking the $L^{2}$ inner product of (3.5) with $\phi$, of (3.6) with $\epsilon U$, and taking the simple multiplication of (3.7) with $M_{1} V$, of (3.8) with $M_{2} W$, and then summing them up, we can obtain the energy dissipation law of the new system (3.5)-(3.8) as

$$
\frac{d}{d t} \mathbb{E}(U, V, W)=-\left\|\phi_{t}\right\|^{2} \leq 0
$$

Remark 3.1. We emphasize that the new transformed system (3.5)-(3.8) is exactly equivalent to the original system (2.6), since (3.1)-(3.3) can be easily obtained by integrating (3.6)-(3.8) with respect to the time. For the time-continuous case, the potentials in the new free energy (3.4) are the same as the Lyapunov functional in the original free energy of (2.5), and the new energy law (3.10) for the transformed system is also the same as the energy law (2.7) for the original system as well. We will develop unconditionally energy stable numerical schemes for time stepping of the transformed system (3.5)-(3.8), and the proposed schemes should formally 
follow the new energy dissipation law (3.10) in the discrete sense, instead of the energy law for the originated system (2.7).

The time marching numerical schemes are developed to solve the new transformed system (3.5)-(3.8). The proof of the unconditional stability of the schemes follow the similar lines as in the derivation of the energy law (3.10). Let $\delta t>0$ denote the time step size and set $t^{n}=n \delta t$ for $0 \leq n \leq N$ with the ending time $T=N \delta t$.

3.2. First order scheme. We now present the first order time stepping scheme to solve the system (3.5)-(3.8). Assumming that $\phi^{n}, U^{n}, V^{n}, W^{n}$ are already calculated, we then compute $\phi^{n+1}, U^{n+1}, V^{n+1}$ and $W^{n+1}$ from the following temporally discretized system:

$$
\begin{aligned}
\frac{\phi^{n+1}-\phi^{n}}{\delta t} & =-\epsilon\left(\Delta-f^{\prime}\left(\phi^{n}\right)\right) U^{n+1}-M_{1} V^{n+1}-\epsilon M_{2} W^{n+1}\left(-\Delta \phi^{n}+f\left(\phi^{n}\right)\right), \\
U^{n+1}-U^{n} & =\left(\Delta-f^{\prime}\left(\phi^{n}\right)\right)\left(\phi^{n+1}-\phi^{n}\right), \\
V^{n+1}-V^{n} & =\int_{\Omega}\left(\phi^{n+1}-\phi^{n}\right) d \boldsymbol{x} \\
W^{n+1}-W^{n} & =\int_{\Omega} \epsilon\left(-\Delta \phi^{n}+f\left(\phi^{n}\right)\right)\left(\phi^{n+1}-\phi^{n}\right) d \boldsymbol{x}
\end{aligned}
$$

with the periodic boundary condition (or the no-flux boundary condition).

We note that (3.12), (3.13) and (3.14) can be rewritten as

$$
\begin{aligned}
& U^{n+1}=A_{1}+A_{2}\left(\phi^{n+1}\right), \\
& V^{n+1}=A_{3}+A_{4}\left(\phi^{n+1}\right), \\
& W^{n+1}=A_{5}+A_{6}\left(\phi^{n+1}\right),
\end{aligned}
$$

where

$$
\begin{cases}A_{1}=U^{n}-\left(\Delta-f^{\prime}\left(\phi^{n}\right)\right) \phi^{n}, & A_{2}(\phi)=\left(\Delta-f^{\prime}\left(\phi^{n}\right)\right) \phi, \\ A_{3}=V^{n}-\int_{\Omega} \phi^{n} d \boldsymbol{x}, & A_{4}(\phi)=\int_{\Omega} \phi d \boldsymbol{x}, \\ A_{5}=W^{n}-\int_{\Omega} \epsilon\left(-\Delta \phi^{n}+f\left(\phi^{n}\right)\right) \phi^{n} d \boldsymbol{x}, & A_{6}(\phi)=\int_{\Omega} \epsilon\left(-\Delta \phi^{n}+f\left(\phi^{n}\right)\right) \phi d \boldsymbol{x} .\end{cases}
$$

Thus (3.11) can be rewritten as the following reduced linear system:

$$
\begin{gathered}
\frac{1}{\delta t} \phi^{n+1}+\epsilon\left(\Delta-f^{\prime}\left(\phi^{n}\right)\right) A_{2}\left(\phi^{n+1}\right)+M_{1} A_{4}\left(\phi^{n+1}\right)+\epsilon M_{2}\left(-\Delta \phi^{n}+f\left(\phi^{n}\right)\right) A_{6}\left(\phi^{n+1}\right) \\
=\frac{1}{\delta t} \phi^{n}-\epsilon A_{2}\left(A_{1}\right)-M_{1} A_{3}-\epsilon M_{2}\left(-\Delta \phi^{n}+f\left(\phi^{n}\right)\right) A_{5} .
\end{gathered}
$$

Let us express the above linear system (3.18) as $\mathbb{A} \phi^{n+1}=b$ and we need solve for $\phi^{n+1}$ from it.

Theorem 3.1. The linear operator $\mathbb{A}$ is symmetric (self-adjoint) positive definite and the linear system (3.18) admits a unique solution. 
Proof. We can easily derive

$$
\begin{aligned}
(\mathbb{A} \phi, \psi)= & \frac{1}{\delta t}(\phi, \psi)+\left(\epsilon\left(\Delta-f^{\prime}\left(\phi^{n}\right)\right) A_{2}(\phi), \psi\right) \\
& +M_{1}\left(A_{4}(\phi), \psi\right)+\left(\epsilon M_{2}\left(-\Delta \phi^{n}+f\left(\phi^{n}\right)\right) A_{6}(\phi), \psi\right) \\
= & \frac{1}{\delta t}(\phi, \psi)+\epsilon\left(A_{2}(\phi), A_{2}(\psi)\right)+M_{1} A_{4}(\phi) A_{4}(\psi)+M_{2} A_{6}(\phi) A_{6}(\psi) \\
= & (\phi, \mathbb{A} \psi),
\end{aligned}
$$

and

$$
(\mathbb{A} \phi, \phi) \geq \frac{1}{\delta t}\|\phi\|^{2}
$$

Thus the operator $\mathbb{A}$ is symmetric positive definite and the linear system (3.18) admits a unique solution.

Therefore we can first solve $\phi^{n+1}$ directly from the linear system (3.18). Once $\phi^{n+1}$ is found, we can readily compute $U^{n+1}, V^{n+1}, W^{n+1}$ from (3.15)-(3.17). Namely, the new auxiliary variables $U, V, W$ do not add any extra computational complexity.

The stability result of the proposed first order scheme is given and proved below.

Theorem 3.2. The first order linear scheme (3.11)-(3.14) is unconditionally energy stable, i.e., satisfies the following discrete energy dissipation law:

$$
E_{1 s t}^{n+1} \leq E_{1 s t}^{n}-\delta t\left\|\frac{\phi^{n+1}-\phi^{n}}{\delta t}\right\|^{2}
$$

where

$$
E_{1 s t}^{n}\left(U^{n}, V^{n}, W^{n}\right)=\frac{\eta}{2}\left\|U^{n}\right\|^{2}+\frac{M_{1}}{2}\left(V^{n}\right)^{2}+\frac{M_{2}}{2}\left(W^{n}\right)^{2} .
$$

Proof. By taking the $L^{2}$ inner product of (3.11) with $\phi^{n+1}-\phi^{n}$, we obtain

$$
\begin{aligned}
\frac{1}{\delta t}\left\|\phi^{n+1}-\phi^{n}\right\|^{2}= & -\epsilon\left(\left(\Delta-f^{\prime}\left(\phi^{n}\right)\right) U^{n+1}, \phi^{n+1}-\phi^{n}\right)-M_{1}\left(V^{n+1}, \phi^{n+1}-\phi^{n}\right) \\
& -\epsilon M_{2}\left(W^{n+1}\left(-\Delta \phi^{n}+f\left(\phi^{n}\right)\right), \phi^{n+1}-\phi^{n}\right) .
\end{aligned}
$$

By taking the $L^{2}$ inner product of (3.12) with $\epsilon U^{n+1}$ and applying the following identities

$$
2(a-b, a)=|a|^{2}-|b|^{2}+|a-b|^{2},
$$

we obtain

$$
\frac{\epsilon}{2}\left(\left\|U^{n+1}\right\|^{2}-\left\|U^{n}\right\|^{2}+\left\|U^{n+1}-U^{n}\right\|^{2}\right)=\epsilon\left(\left(\Delta-f^{\prime}\left(\phi^{n}\right)\right)\left(\phi^{n+1}-\phi^{n}\right), U^{n+1}\right) .
$$

By taking the simple multiplication of (3.13) with $M_{1} V^{n+1}$ and applying (3.24), we obtain

$$
\frac{M_{1}}{2}\left(\left(V^{n+1}\right)^{2}-\left(V^{n}\right)^{2}+\left(V^{n+1}-V^{n}\right)^{2}\right)=M_{1}\left(\phi^{n+1}-\phi^{n}, V^{n+1}\right) .
$$

By taking the simple multiplication of (3.14) with $M_{2} W^{n+1}$ and applying (3.24), we obtain

$$
\begin{aligned}
\frac{M_{2}}{2}\left(\left(W^{n+1}\right)^{2}\right. & \left.-\left(W^{n}\right)^{2}+\left(W^{n+1}-W^{n}\right)^{2}\right) \\
& =\epsilon M_{2}\left(\left(-\Delta \phi^{n}+f\left(\phi^{n}\right)\right)\left(\phi^{n+1}-\phi^{n}\right), W^{n+1}\right) .
\end{aligned}
$$


Combination of (3.23), (3.25)-(3.27) gives us

$$
\begin{gathered}
\frac{\epsilon}{2}\left(\left\|U^{n+1}\right\|^{2}-\left\|U^{n}\right\|^{2}+\left\|U^{n+1}-U^{n}\right\|^{2}\right)+\frac{M_{1}}{2}\left(\left(V^{n+1}\right)^{2}-\left(V^{n}\right)^{2}+\left(V^{n+1}-V^{n}\right)^{2}\right) \\
+\frac{M_{2}}{2}\left(\left(W^{n+1}\right)^{2}-\left(W^{n}\right)^{2}+\left(W^{n+1}-W^{n}\right)^{2}\right)=-\frac{1}{\delta t}\left\|\phi^{n+1}-\phi^{n}\right\|^{2} .
\end{gathered}
$$

Finally, we obtain the desired result (3.21) after dropping some positive terms from (3.28).

Remark 3.2. The proposed schemes follow the new energy dissipation law (3.10) instead of the energy law for the originated system (2.7). In the time-discrete case, the energy $E_{1 s t}\left(U^{n+1}, V^{n+1}, W^{n+1}\right)$ (defined in (3.22)) can be rewritten as a first order approximation to the Lyapunov functionals as $E\left(\phi^{n+1}\right)$ (defined in (2.5)), that can be observed from the following facts, heuristically. From (3.12) we have

$$
U^{n+1}-\left(\Delta \phi^{n+1}-f\left(\phi^{n+1}\right)\right)=U^{n}-\left(\Delta \phi^{n}-f\left(\phi^{n}\right)\right)+R_{n+1},
$$

where

$$
R_{n+1}=f\left(\phi^{n+1}\right)-f\left(\phi^{n}\right)-f^{\prime}\left(\phi^{n}\right)\left(\phi^{n+1}-\phi^{n}\right) .
$$

Since $R_{k}=O\left(\delta t^{2}\right)$ for $0 \leq k \leq n+1$ and $U^{0}=\Delta \phi^{0}-f\left(\phi^{0}\right)$, by mathematical induction we can easily get $U^{n+1}=\Delta \phi^{n+1}-f\left(\phi^{n+1}\right)+O(\delta t)$. The same argument can be performed for the variables $V^{n+1}$ and $W^{n+1}$.

Remark 3.3. The essential idea of the IEQ method is to transform the complicated nonlinear potentials into a simple quadratic form in terms of some new variables via a change of variables. Such simple way of quadratization provides some great advantages. First, the complicated nonlinear potential is transferred to quadratic polynomial form that is much easier to handle. Second, the derivative of the quadratic polynomial is linear, which provides the fundamental support for linearization method. Third, the quadratic formulation in terms of new variables can automatically keep this property of positivity (or bounded from below) of the nonlinear potentials. Overall, the IEQ approach provides many flexibilities to treat the complicated nonlinear terms, for instances, the nonlinear potential only need to be positive, or bounded from below, instead of the convexity that is required in the convex splitting approach.

3.3. Second order scheme. Now we construct the second order time stepping scheme based on the Adam-Bashforth backward differentiation formulas (BDF2). Assuming that we have found $\phi^{n-1}, U^{n-1}, V^{n-1}, W^{n-1}$ and $\phi^{n}, U^{n}, V^{n}, W^{n}$, then we compute $\phi^{n+1}, U^{n+1}, V^{n+1}$, $W^{n+1}$ as follows:

$$
\begin{aligned}
\frac{3 \phi^{n+1}-4 \phi^{n}+\phi^{n-1}}{2 \delta t}= & -\epsilon\left(\Delta-f^{\prime}\left(\phi^{\star}\right)\right) U^{n+1} \\
& -M_{1} V^{n+1}-\epsilon M_{2} W^{n+1}\left(-\Delta \phi^{\star}+f\left(\phi^{\star}\right)\right), \\
3 U^{n+1}-4 U^{n}+U^{n-1}= & \left(\Delta-f^{\prime}\left(\phi^{\star}\right)\right)\left(3 \phi^{n+1}-4 \phi^{n}+\phi^{n-1}\right), \\
3 V^{n+1}-4 V^{n}+V^{n-1}= & \int_{\Omega}\left(3 \phi^{n+1}-4 \phi^{n}+\phi^{n-1}\right) d \boldsymbol{x}, \\
3 W^{n+1}-4 W^{n}+W^{n-1}= & \int_{\Omega} \epsilon\left(-\Delta \phi^{\star}+f\left(\phi^{\star}\right)\right)\left(3 \phi^{n+1}-4 \phi^{n}+\phi^{n-1}\right) d \boldsymbol{x}
\end{aligned}
$$

with the periodic boundary condition (or the no-flux boundary condition) and $\phi^{\star}=2 \phi^{n}-\phi^{n-1}$. 
Note that (3.32), (3.33) and (3.34) can be rewritten as

$$
\begin{aligned}
& U^{n+1}=B_{1}+B_{2}\left(\phi^{n+1}\right), \\
& V^{n+1}=B_{3}+B_{4}\left(\phi^{n+1}\right), \\
& W^{n+1}=B_{5}+B_{6}\left(\phi^{n+1}\right),
\end{aligned}
$$

where

$$
\begin{cases}B_{1}=U^{\dagger}-\left(\Delta-f^{\prime}\left(\phi^{\star}\right)\right) \phi^{\dagger}, & B_{2}(\phi)=\left(\Delta-f^{\prime}\left(\phi^{\star}\right)\right) \phi \\ B_{3}=V^{\dagger}-\int_{\Omega} \phi^{\dagger} d \boldsymbol{x}, & B_{4}(\phi)=\int_{\Omega} \phi d \boldsymbol{x} \\ B_{5}=W^{\dagger}-\int_{\Omega} \epsilon\left(-\Delta \phi^{\star}+f\left(\phi^{\star}\right)\right) \phi^{\dagger} d \boldsymbol{x}, & B_{6}(\phi)=\int_{\Omega} \epsilon\left(-\Delta \phi^{\star}+f\left(\phi^{\star}\right)\right) \phi d \boldsymbol{x}\end{cases}
$$

with $Q^{\dagger}=\frac{4 Q^{n}-Q^{n-1}}{3}$ for any variable $Q$. In turn, (3.31) can be rewritten as the following reduced linear system:

$$
\begin{aligned}
\frac{3}{2 \delta t} \phi^{n+1} & +\epsilon\left(\Delta-f^{\prime}\left(\phi^{\star}\right)\right) B_{2}\left(\phi^{n+1}\right)+M_{1} B_{4}\left(\phi^{n+1}\right)+\epsilon M_{2}\left(-\Delta \phi^{\star}+f\left(\phi^{\star}\right)\right) B_{6}\left(\phi^{n+1}\right) \\
& =\frac{1}{2 \delta t}\left(4 \phi^{n}-\phi^{n-1}\right)-\epsilon B_{2}\left(B_{1}\right)-M_{1} B_{3}-\epsilon M_{2}\left(-\Delta \phi^{\star}+f\left(\phi^{\star}\right)\right) B_{5} .
\end{aligned}
$$

Let us express the above linear system (3.38) as $\widehat{\mathbb{A}} \phi^{n+1}=\widehat{b}$ and we need solve for $\phi^{n+1}$ from it. Similar to the first order scheme, we can obtain the following result.

Theorem 3.3. The linear operator $\widehat{\mathbb{A}}$ is symmetric (self-adjoint) positive definite and the linear system (3.38) admits a unique solution.

Proof. It is not hard to find that

$$
\begin{aligned}
(\widehat{\mathbb{A}} \phi, \psi) & =\frac{2}{2 \delta t}(\phi, \psi)+\epsilon\left(B_{2}(\phi), B_{2}(\psi)\right)+M_{1} B_{4}(\phi) B_{4}(\psi)+M_{2} B_{6}(\phi) B_{6}(\psi) \\
& =(\phi, \widehat{\mathbb{A}} \psi),
\end{aligned}
$$

and

$$
(\mathbb{A} \phi, \phi) \geq \frac{3}{2 \delta t}\|\phi\|^{2} .
$$

Thus the operator $\mathbb{A}$ is symmetric positive definite and the linear system (3.38) admits a unique solution.

Therefore, we again can first solve $\phi^{n+1}$ directly from (3.38). Once $\phi^{n+1}$ are obtained, we can readily compute $U^{n+1}, V^{n+1}$ and $W^{n+1}$ from (3.35)-(3.37).

Theorem 3.4. The second order linear scheme (3.31)-(3.34) is unconditionally energy stable, i.e., satisfies the following discrete energy dissipation law:

$$
E_{2}^{n+1, n} \leq E_{2}^{n, n-1}-\delta t\left\|\frac{3 \phi^{n+1}-4 \phi^{n}+\phi^{n-1}}{2 \delta t}\right\|^{2},
$$


where

$$
\begin{aligned}
E_{2}^{n+1, n} & =\frac{\epsilon}{4}\left(\left\|U^{n+1}\right\|^{2}+\left\|2 U^{n+1}-U^{n}\right\|^{2}\right)+\frac{M_{1}}{4}\left(\left(V^{n+1}\right)^{2}+\left(2 V^{n+1}-V^{n}\right)^{2}\right) \\
& +\frac{M_{2}}{4}\left(\left(W^{n+1}\right)^{2}+\left(2 W^{n+1}-W^{n}\right)^{2}\right) .
\end{aligned}
$$

Proof. By taking the $L^{2}$ inner product of (3.31) with $\left(3 \phi^{n+1}-4 \phi^{n}+\phi^{n-1}\right)$, we obtain

$$
\begin{aligned}
\frac{1}{2 \delta t}\left\|3 \phi^{n+1}-4 \phi^{n}+\phi^{n-1}\right\|^{2}= & -\epsilon\left(\left(\Delta-f^{\prime}\left(\phi^{\star}\right)\right) U^{n+1}, 3 \phi^{n+1}-4 \phi^{n}+\phi^{n-1}\right) \\
& -M_{1}\left(V^{n+1}, 3 \phi^{n+1}-4 \phi^{n}+\phi^{n-1}\right) \\
& -\epsilon M_{2}\left(W^{n+1},\left(-\Delta \phi^{\star}+f\left(\phi^{\star}\right)\right), 3 \phi^{n+1}-4 \phi^{n}+\phi^{n-1}\right) .
\end{aligned}
$$

By taking the $L^{2}$ inner product of (3.32) with $\epsilon U^{n+1}$ and applying the following identity

$$
2(3 a-4 b+c, a)=|a|^{2}-|b|^{2}+|2 a-b|^{2}-|2 b-c|^{2}+|a-2 b+c|^{2},
$$

we obtain

$$
\begin{aligned}
& \frac{\epsilon}{2}\left(\left\|U^{n+1}\right\|^{2}-\left\|U^{n}\right\|^{2}+\left\|2 U^{n+1}-U^{n}\right\|^{2}-\left\|U^{n}-U^{n-1}\right\|^{2}\right. \\
& \left.\quad+\left\|U^{n+1}-2 U^{n}+U^{n-1}\right\|^{2}\right)=\epsilon\left(\left(\Delta-f^{\prime}\left(\phi^{\star}\right)\right)\left(3 \phi^{n+1}-4 \phi^{n}+\phi^{n-1}\right), U^{n+1}\right) .
\end{aligned}
$$

By taking the simple multiplication of (3.33) with $M_{1} V^{n+1}$, we obtain

$$
\begin{aligned}
& \frac{M_{1}}{2}\left(\left(V^{n+1}\right)^{2}-\left(V^{n}\right)^{2}+\left(2 V^{n+1}-V^{n}\right)^{2}-\left(2 V^{n}-V^{n-1}\right)^{2}\right. \\
& \left.\quad+\left(V^{n+1}-2 V^{n}+V^{n-1}\right)^{2}\right)=M_{1}\left(3 \phi^{n+1}-4 \phi^{n}+\phi^{n-1}, V^{n+1}\right) .
\end{aligned}
$$

By taking the simple multiplication of (3.34) with $M_{2} W^{n+1}$, we obtain

$$
\begin{aligned}
& \frac{M_{2}}{2}\left(\left(W^{n+1}\right)^{2}-\left(W^{n+1}\right)^{2}+\left(2 W^{n+1}-W^{n}\right)^{2}-\left(2 W^{n}-W^{n-1}\right)^{2}\right. \\
& \left.+\left(W^{n+1}-2 W^{n}+W^{n-1}\right)^{2}\right)=\epsilon M_{2}\left(\left(-\Delta \phi^{\star}+f\left(\phi^{\star}\right)\right)\left(3 \phi^{n+1}-4 \phi^{n}+\phi^{n-1}\right), W^{n+1}\right)
\end{aligned}
$$

Combination of (3.42), and (3.44)-(3.46) gives us

$$
\begin{aligned}
& \frac{\epsilon}{2}\left(\left\|U^{n+1}\right\|^{2}-\left\|U^{n}\right\|^{2}+\left\|2 U^{n+1}-U^{n}\right\|^{2}-\left\|2 U^{n}-U^{n-1}\right\|^{2}+\left\|U^{n+1}-2 U^{n}+U^{n-1}\right\|^{2}\right) \\
& \quad+\frac{M_{1}}{2}\left(\left(V^{n+1}\right)^{2}-\left(V^{n}\right)^{2}+\left(2 V^{n+1}-V^{n}\right)^{2}-\left(2 V^{n}-V^{n-1}\right)^{2}+\left(V^{n+1}-2 V^{n}+V^{n-1}\right)^{2}\right) \\
& \quad+\frac{M_{2}}{2}\left(\left(W^{n+1}\right)^{2}-\left(W^{n+1}\right)^{2}+\left(2 W^{n+1}-W^{n}\right)^{2}-\left(2 W^{n}-W^{n-1}\right)^{2}+\left(W^{n+1}-2 W^{n}+W^{n-1}\right)^{2}\right) \\
& \quad=-\frac{1}{2 \delta t}\left\|3 \phi^{n+1}-4 \phi^{n}+\phi^{n-1}\right\|^{2} .
\end{aligned}
$$

Finally, we obtain the desired result (3.41) after dropping some positive terms from the above equation. 
Remark 3.4. Heuristically, the discrete energy dissipation law (3.41) is a second order approximation of $\frac{d}{d t} E(\phi)$ in (3.4) since

$$
\begin{aligned}
& \left(\frac{\left\|V^{n+1}\right\|^{2}+\left\|2 V^{n+1}-V^{n}\right\|^{2}}{2 \delta t}\right)-\left(\frac{\left\|V^{n}\right\|^{2}+\left\|2 V^{n}-V^{n-1}\right\|^{2}}{2 \delta t}\right) \\
& =\left(\frac{\left\|V^{n+2}\right\|^{2}-\left\|V^{n}\right\|^{2}}{2 \delta t}\right)+O\left(\delta t^{2}\right)=\frac{d}{d t}\left\|V\left(t^{n+1}\right)\right\|^{2}+O\left(\delta t^{2}\right) .
\end{aligned}
$$

for any smooth variable $V$.

Remark 3.5. It is also straightforward to develop the second order Crank-Nicolson scheme. Thus the details are left to the interested readers. In addition, although we consider only time discrete schemes in this study, the results can be carried over to any consistent finite-dimensional Galerkin approximations in the space since the proofs are all based on a variational formulation with all test functions in the same space as the space of the trial functions.

Remark 3.6. The local truncation error of the proposed schemes can be readily derived via Taylor series expansion assuming that the classical solutions of the PDE system have enough regularity. We omit the details here for brevity. The complete error analysis of the schemes will be pursued in a separate article.

\section{NumericAl EXPERIMENTS}

We now present various numerical experiments to validate the theoretical results derived in the previous sections and demonstrate the stability and accuracy of the proposed numerical schemes. In all examples, we set the domain $\Omega=[0,2 \pi]^{d}, d=2,3$. We use the Fourier-spectral method to discretize the space where $129 \times 129$ Fourier modes are used for 2D simulations, and $129 \times 129 \times 129$ Fourier modes are used for 3 D simulations. We set $\epsilon=3 h$ where $h=\frac{2 \pi}{128}$ throughout all simulations.

4.1. Accuracy test. We first perform numerical simulations to test the convergence rates of the two proposed schemes, the first order linear scheme (3.11)-(3.14) (denoted by LS1) and the second order linear scheme (3.31)-(3.34) (denoted by LS2).

4.1.1. Presumed exact solution. In the first example, we assume the following function

$$
\phi(x, y, t)=\sin (x) \sin (y) \sin (t)
$$

to be the exact solution, and impose some suitable force fields such that the given solution can satisfy the system (2.6). The two penalty parameters for fixed volume and surface area constraints are set to be $M_{1}=M_{2}=50$. In Table 4.1, we list the $L^{2}$ errors of the phase variable $\phi$ between the numerically simulated solution and the exact solution at $t=0.1$ with different time step sizes. We observe that the schemes LS1 and LS2 achieve almost perfect first-order and second order accuracy in time, respectively.

4.1.2. More temporal convergence tests. We perform more tests for demonstrating temporal convergence of the proposed schemes in $2 \mathrm{D}$ and $3 \mathrm{D}$. The initial condition of $\phi$ is set to be two 


\begin{tabular}{|r||c|c|c|c|}
\hline$\delta t$ & LS1 & Order & LS2 & Order \\
\hline \hline $2 \times 10^{-2}$ & $6.53 \mathrm{E}-3$ & - & $1.01 \mathrm{E}-3$ & - \\
\hline $1 \times 10^{-2}$ & $3.07 \mathrm{E}-3$ & 1.09 & $2.34 \mathrm{E}-4$ & 2.10 \\
\hline $5 \times 10^{-3}$ & $1.49 \mathrm{E}-3$ & 1.04 & $5.68 \mathrm{E}-5$ & 2.04 \\
\hline $1 \times 10^{-3}$ & $2.92 \mathrm{E}-4$ & 1.01 & $2.25 \mathrm{E}-6$ & 2.01 \\
\hline $5 \times 10^{-4}$ & $1.45 \mathrm{E}-4$ & 1.01 & $5.66 \mathrm{E}-7$ & 1.99 \\
\hline $1 \times 10^{-4}$ & $2.91 \mathrm{E}-5$ & 1.00 & $2.25 \mathrm{E}-8$ & 2.00 \\
\hline
\end{tabular}

TABLE 4.1. The $L^{2}$ errors at $t=0.1$ for the approximate phase variable $\phi$ of (2.6) with the penalty parameters $M_{1}=M_{2}=50$ and the presumed exact solution (4.1) in 2D, computed by the schemes LS1 and LS2 using various temporal resolutions. Note the time step size is not necessary to be halved along the time step size refinement since the exact solution is known.

\begin{tabular}{|r||c|c|c|c|}
\hline$\delta t$ & LS1 & Order & LS2 & Order \\
\hline \hline $1 \times 10^{-2}$ & $2.47 \mathrm{E}-3$ & - & $2.30 \mathrm{E}-3$ & - \\
\hline $5 \times 10^{-3}$ & $1.32 \mathrm{E}-3$ & 0.90 & $1.20 \mathrm{E}-3$ & 0.94 \\
\hline $2.5 \times 10^{-3}$ & $7.06 \mathrm{E}-4$ & 0.90 & $6.49 \mathrm{E}-4$ & 0.89 \\
\hline $1.25 \times 10^{-3}$ & $3.76 \mathrm{E}-4$ & 0.91 & $3.17 \mathrm{E}-4$ & 1.03 \\
\hline $6.25 \times 10^{-4}$ & $1.98 \mathrm{E}-4$ & 0.93 & $1.31 \mathrm{E}-4$ & 1.27 \\
\hline $3.125 \times 10^{-4}$ & $1.01 \mathrm{E}-4$ & 0.97 & $4.56 \mathrm{E}-5$ & 1.52 \\
\hline $1.5625 \times 10^{-4}$ & $5.04 \mathrm{E}-5$ & 1.00 & $1.17 \mathrm{E}-5$ & 1.96 \\
\hline $7.8125 \times 10^{-5}$ & $2.49 \mathrm{E}-5$ & 1.02 & $3.02 \mathrm{E}-6$ & 1.95 \\
\hline $3.90625 \times 10^{-5}$ & $1.23 \mathrm{E}-5$ & 1.01 & $7.49 \mathrm{E}-7$ & 2.01 \\
\hline
\end{tabular}

TABLE 4.2. The $L^{2}$ errors at $t=0.1$ for the approximate phase variable $\phi$ of (2.6) with the initial configuration (4.2) and $M_{1}=M_{2}=50$ in 2D, computed by the schemes LS1 and LS2 using various temporal resolutions.

close-by spherical vesicles in $2 \mathrm{D}$ as

$$
\begin{aligned}
\phi(x, y, 0)= & \tanh \left(\frac{0.28 \pi-\sqrt{x^{2}+(y+0.35 \pi)^{2}}}{\sqrt{2} \epsilon}\right) \\
& +\tanh \left(\frac{0.28 \pi-\sqrt{x^{2}+(y-0.35 \pi)^{2}}}{\sqrt{2} \epsilon}\right)+1,
\end{aligned}
$$




\begin{tabular}{|r||c|c|c|c|}
\hline$\delta t$ & LS1 & Order & LS2 & Order \\
\hline \hline $1 \times 10^{-2}$ & $4.39 \mathrm{E}-3$ & - & $3.81 \mathrm{E}-3$ & - \\
\hline $5 \times 10^{-3}$ & $2.22 \mathrm{E}-3$ & 0.95 & $1.69 \mathrm{E}-3$ & 1.17 \\
\hline $2.5 \times 10^{-3}$ & $1.11 \mathrm{E}-3$ & 1.00 & $6.72 \mathrm{E}-4$ & 1.33 \\
\hline $1.25 \times 10^{-3}$ & $5.53 \mathrm{E}-4$ & 1.01 & $2.60 \mathrm{E}-4$ & 1.37 \\
\hline $6.25 \times 10^{-4}$ & $2.78 \mathrm{E}-4$ & 0.99 & $9.47 \mathrm{E}-5$ & 1.46 \\
\hline $3.125 \times 10^{-4}$ & $1.39 \mathrm{E}-4$ & 1.00 & $3.04 \mathrm{E}-5$ & 1.64 \\
\hline $1.5625 \times 10^{-4}$ & $6.94 \mathrm{E}-5$ & 1.00 & $7.49 \mathrm{E}-6$ & 2.02 \\
\hline $7.8125 \times 10^{-5}$ & $3.52 \mathrm{E}-5$ & 0.98 & $1.90 \mathrm{E}-6$ & 1.98 \\
\hline $3.90625 \times 10^{-5}$ & $1.77 \mathrm{E}-5$ & 0.99 & $4.65 \mathrm{E}-7$ & 2.03 \\
\hline
\end{tabular}

TABLE 4.3. The $L^{2}$ errors at $t=0.1$ for the approximate phase variable $\phi$ of (2.6) with the initial configuration (4.3) and $M_{1}=M_{2}=0$ in $3 \mathrm{D}$, computed by the schemes LS1 and LS2 using various temporal resolutions.

\begin{tabular}{|r||c|c|c|c|}
\hline$\delta t$ & LS1 & Order & LS2 & Order \\
\hline \hline $1 \times 10^{-2}$ & $4.10 \mathrm{E}-3$ & - & $3.56 \mathrm{E}-3$ & - \\
\hline $5 \times 10^{-3}$ & $2.17 \mathrm{E}-3$ & 0.92 & $1.46 \mathrm{E}-3$ & 1.28 \\
\hline $2.5 \times 10^{-3}$ & $1.09 \mathrm{E}-3$ & 0.99 & $5.44 \mathrm{E}-4$ & 1.42 \\
\hline $1.25 \times 10^{-3}$ & $5.51 \mathrm{E}-4$ & 0.98 & $2.00 \mathrm{E}-4$ & 1.44 \\
\hline $6.25 \times 10^{-4}$ & $2.78 \mathrm{E}-4$ & 0.99 & $7.03 \mathrm{E}-5$ & 1.51 \\
\hline $3.125 \times 10^{-4}$ & $1.39 \mathrm{E}-4$ & 1.00 & $2.20 \mathrm{E}-5$ & 1.68 \\
\hline $1.5625 \times 10^{-4}$ & $6.99 \mathrm{E}-5$ & 0.99 & $5.37 \mathrm{E}-6$ & 2.03 \\
\hline $7.8125 \times 10^{-5}$ & $3.42 \mathrm{E}-5$ & 1.03 & $1.37 \mathrm{E}-6$ & 1.97 \\
\hline $3.90625 \times 10^{-5}$ & $1.70 \mathrm{E}-5$ & 1.01 & $3.45 \mathrm{E}-7$ & 1.99 \\
\hline
\end{tabular}

TABLE 4.4. The $L^{2}$ errors at $t=0.1$ for the approximate phase variable $\phi$ of (2.6) with the initial configuration (4.3) and $M_{1}=M_{2}=50$ in 3D, computed by the schemes LS1 and LS2 using various temporal resolutions. 


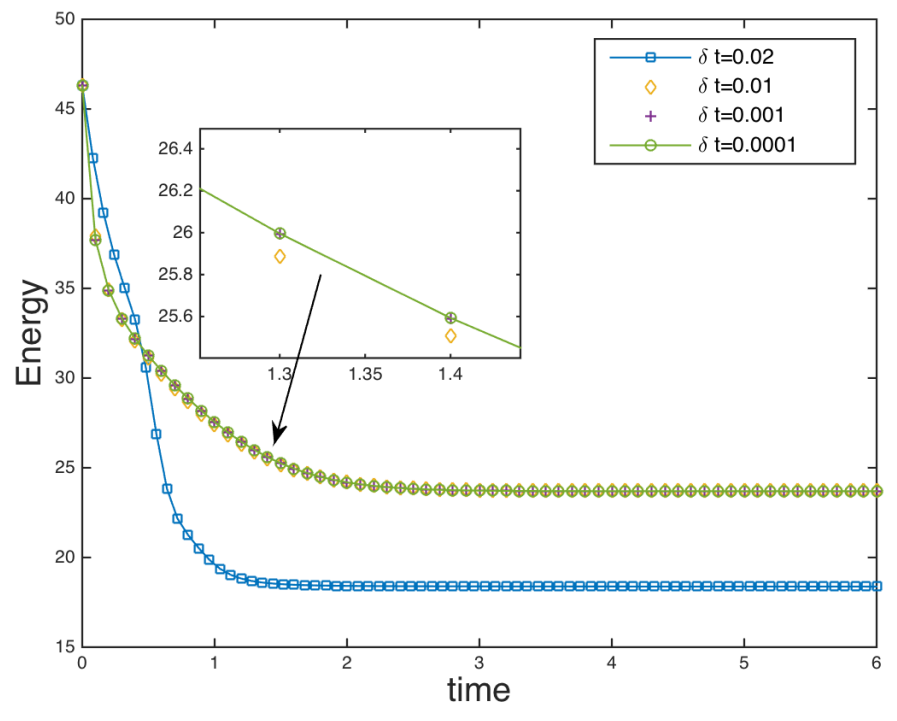

Figure 4.1. Time evolution of the free energy for four different time step sizes, $\delta t=0.0001,0.001,0.01$ and 0.02 for the collision of two 3D close-by spherical vesicles without the volume and surface area constraints (i.e., $M_{1}=M_{2}=0$ ) using the scheme LS2. The small inset figure shows the small differences in the energy evolution for the considered time steps of $\delta t=0.0001,0.001$ and 0.01 .

and in $3 \mathrm{D}$ as

$$
\begin{aligned}
\phi(x, y, z, 0)= & \tanh \left(\frac{0.28 \pi-\sqrt{x^{2}+y^{2}+(z+0.35 \pi)^{2}}}{\sqrt{2} \epsilon}\right) \\
& +\tanh \left(\frac{0.28 \pi-\sqrt{x^{2}+y^{2}+(z-0.35 \pi)^{2}}}{\sqrt{2} \epsilon}\right)+1,
\end{aligned}
$$

respectively.

Since the exact solutions are not known, we choose the solution obtained by the scheme LS2 with the time step size $\delta t=1.953125 \times 10^{-5}$ as the benchmark solution (approximately the exact solution) for computing errors. We present the $L^{2}$ errors of the phase variable between the numerical solution and the benchmark solution at $t=0.1$ with different time step sizes in Table 4.2 for the $2 \mathrm{D}$ case with penalty parameters $M_{1}=M_{2}=50$. In Table 4.3 and Table 4.4, we present the $L^{2}$ errors for $3 \mathrm{D}$ cases with penalty parameters $M_{1}=M_{2}=0$ (i.e., without the volume and surface area constraints) and $M_{1}=M_{2}=50$, respectively. We observe that the schemes LS1 and LS2 asymptotically match the first-order and second order accuracy in time, respectively. Moreover, the second order scheme LS2 gives better and better accuracy along the refinement than the first order scheme LS1 does when using the same time step sizes.

4.2. Collision of two close-by spherical vesicles. In this example, we study the typical transformation (collision) process of the two close-by 3D spherical vesicles with the initial profile given in (4.3). 

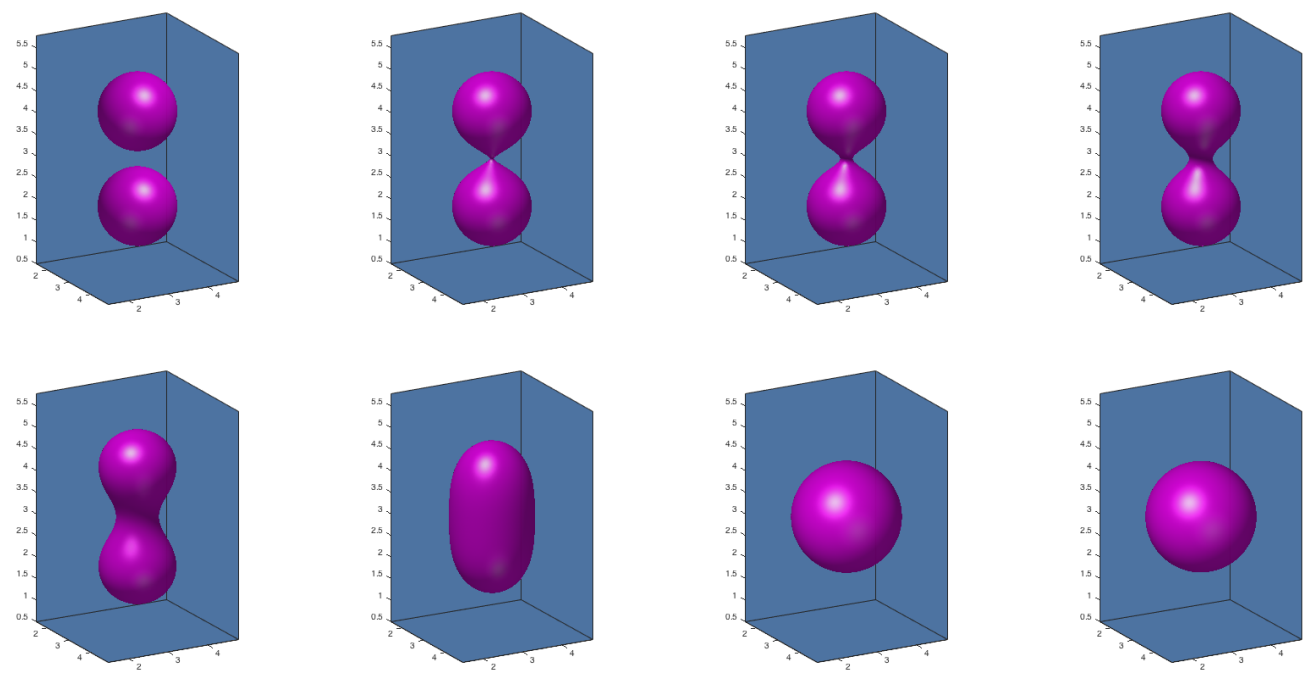

FiguRE 4.2. The dynamical behaviors of the collisions of two 3D close-by spherical vesicles without the volume and surface area constraints $\left(M_{1}=M_{2}=0\right)$ using the scheme LS2 with the time step size $\delta t=0.001$. Snapshots of the numerical approximation of the isosurfaces of $\phi$ are taken at $t=0,0.015,0.018,0.03,0.1$, $1,4,10$.

We first perform the simulation without the volume and surface area constraints (i.e., $M_{1}=$ $\left.M_{2}=0\right)$. We emphasize that any time step size $\delta t$ is allowable for the computations from the stability concern since both the proposed schemes are unconditionally energy stable. But larger time step will definitely induce large numerical errors. Therefore, we need to discover the rough range of the allowable maximum time step size in order to obtain good accuracy and to consume as low computational cost as possible. Such time step range could be estimated through the energy evolution curve plots, shown in Fig. 4.1, where we compare the time evolution of the free energy for four different time step sizes until $t=6$ using the scheme LS2. We observe that all four energy curves show decays monotonically for all time step sizes, which numerically confirms that the proposed schemes are unconditionally energy stable. For smaller time steps of $\delta t=0.0001,0.001,0.01$, the three energy curves coincide very well. But for the larger time step of $\delta t=0.02$, the energy curve deviates much away from others. This means the adopted time step size should not be larger than 0.01 , in order to get reasonably good accuracy. In Fig. 4.2, we show snapshots of the isosurface for the interface $\{\phi(\boldsymbol{x})=0\}$ obtained by the scheme LS2 with the time step size $\delta t=0.001$. The two spheres first merge together and gradually change to a larger sphere, which coincides well with the results discussed in $[14,45]$.

We next perform similar simulations but with the fixed volume and surface area constraints. It can be computed that the fixed scaled volume of the phase variable, $\alpha=V_{S}\left(\phi_{0}\right) \approx 12.83$, and the scaled surface area, $\beta=A_{S}\left(\phi_{0}\right) \approx 18.61$. Note the actual initial volume and surface area are $\frac{1}{2} \alpha=6.42$ and $\frac{3}{2 \sqrt{2}} \beta=19.74$, respectively. We first choose the penalty parameters $M_{1}=M_{2}=50$. In Fig. 4.3, we compare the time evolution of the free energy $E$, the scaled volume difference $V_{S}(\phi)-\alpha$, and the scaled surface area difference $A_{S}(\phi)-\beta$ for four different 

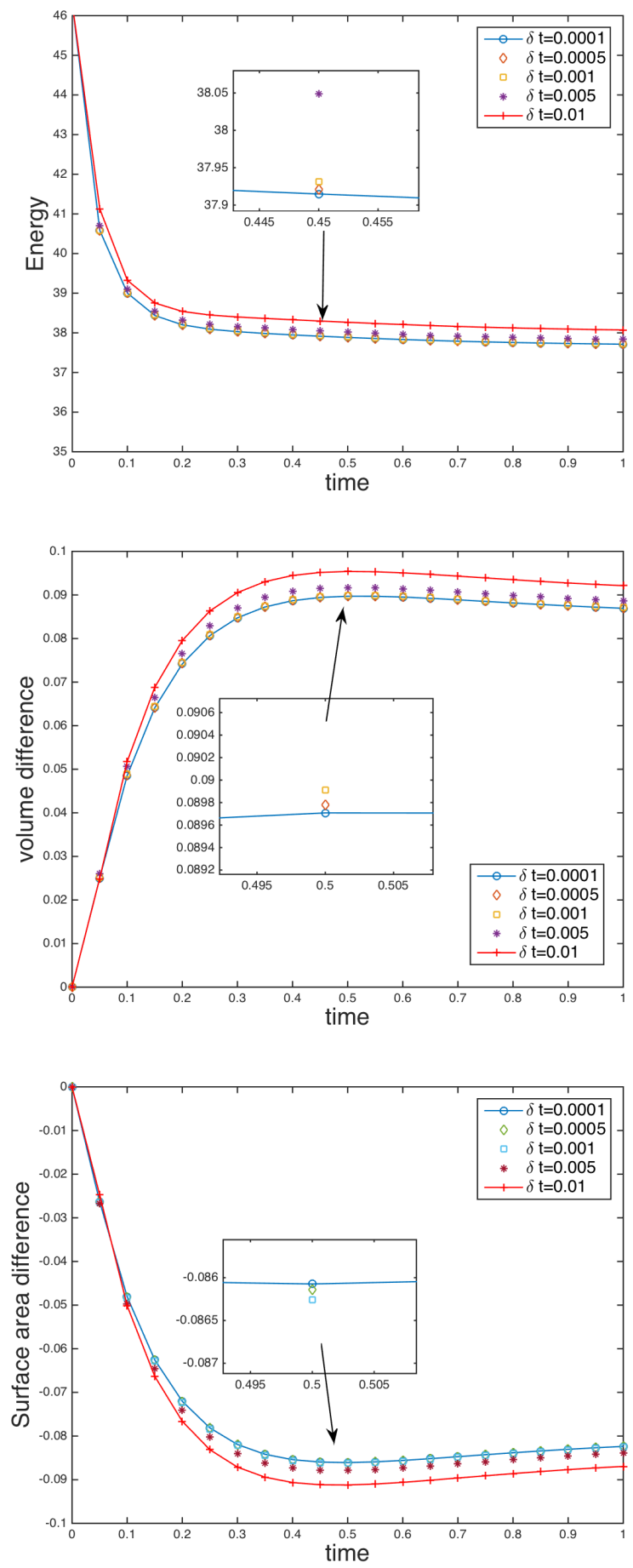

Figure 4.3. Time evolution of the free energy, the scaled volume difference $V_{S}(\phi)-\alpha$, and the scaled surface area difference $A_{S}(\phi)-\beta$ for five different time step sizes, $\delta t=0.0001,0.0005,0.001,0.005$ and 0.01 , for the collision of two 3D close-by spherical vesicles for the case with the volume and surface area constraints $\left(M_{1}=M_{2}=50\right)$ using the second order scheme LS2. 

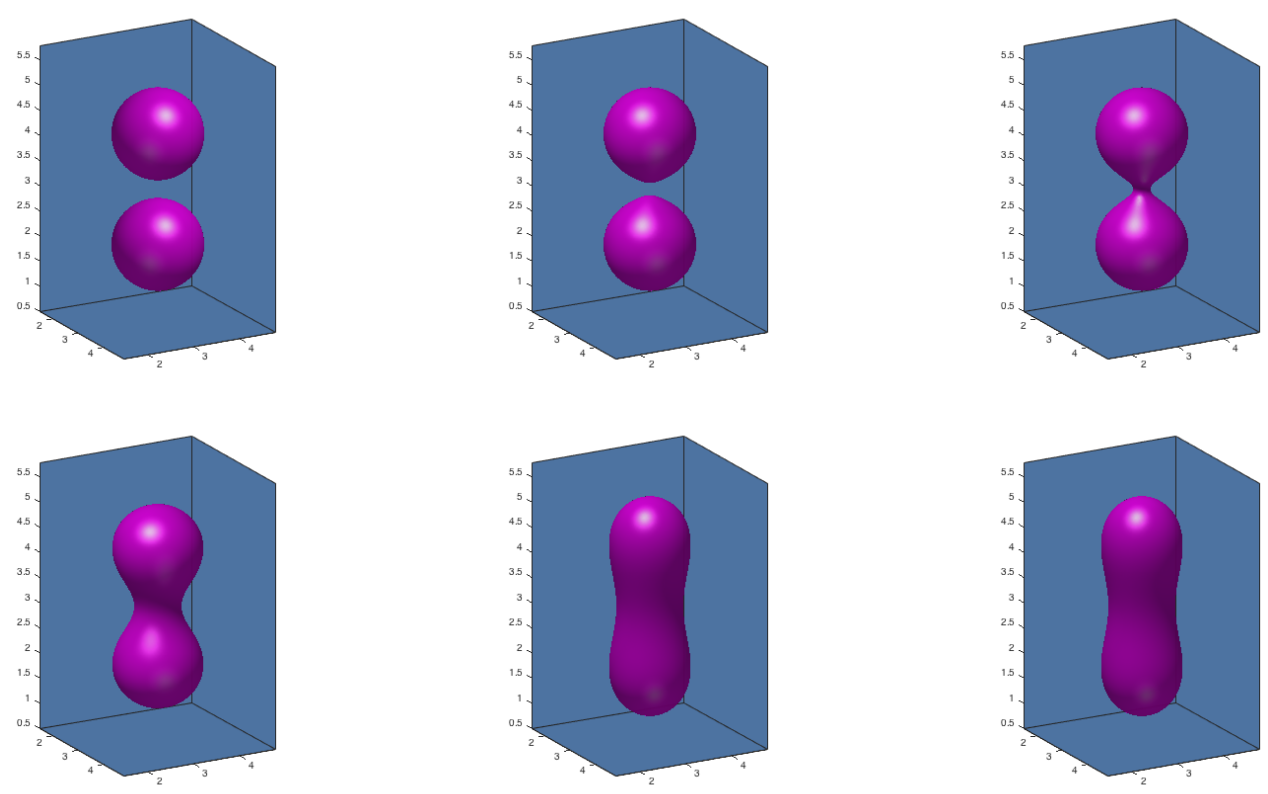

Figure 4.4. The dynamical behaviors of the collision of two 3D close-by spherical vesicles with the surface area and the volume constraints $\left(M_{1}=M_{2}=50\right)$ using the scheme LS2 with the time step size $\delta t=0.001$. Snapshots of the numerical approximation of $\phi$ are taken at $t=0,0.01,0.02,0.1,2,6$.

time step sizes until $t=1$ using the scheme LS2. We notice that the four obtained curves using smaller time steps of $\delta t=0.0001,0.0005,0.001,0.005$ coincide quite well. But when using the larger time step $\delta t=0.01$, the obtained curves deviate considerable away from others. This implies the time step size should be as small as around 0.005 in order to get reasonably good accuracy when using the scheme LS2 for this constrained case. In Fig. 4.4, we show snapshots of the isosurface for the interface obtained by the scheme LS2 with the time step size $\delta t=0.001$. We observe that the two vesicles collide and finally evolve into the capsule shape due to the fixed volume and surface area constraints, which agrees with the results discussed in $[14,45]$. We then further increase the volume and the surface constraint penalty parameters to $M_{1}=M_{2}=1000$. Fig. 4.5 presents the snapshots of the isosurface produced by using the scheme LS2 with the time step size $\delta t=0.001$. We observe that the two vesicles evolve to the similar capsule shape as 4.4. In Fig. 4.6, we plot the comparisons of the time evolution of the free energy, the volume difference, and the surface area difference for the constrained cases with $M_{1}=M_{2}=50$ and $M_{1}=M_{2}=1000$. It can be observed that the fixed volume and the surface area constraints are preserved better when using the larger penalty parameters $M_{1}=M_{2}=1000$ as expected, for instance, the volume difference is around $0.23 \%$ and the surface area difference is around $0.003 \%$ when $M_{1}=M_{2}=1000$ (for $M_{1}=M_{2}=50$, the percentages are $0.7 \%$ and $0.5 \%$ for the volume and surface area, respectively).

One can further reduce this value by increasing the two penalty parameters $M_{1}$ and $M_{2}$. In Fig. 4.7, we show the evolution curves for the energy, the volume difference and the surface area difference produced the constrained model with the penalty parameters $M_{1}=M_{2}=50,500,5000$ 

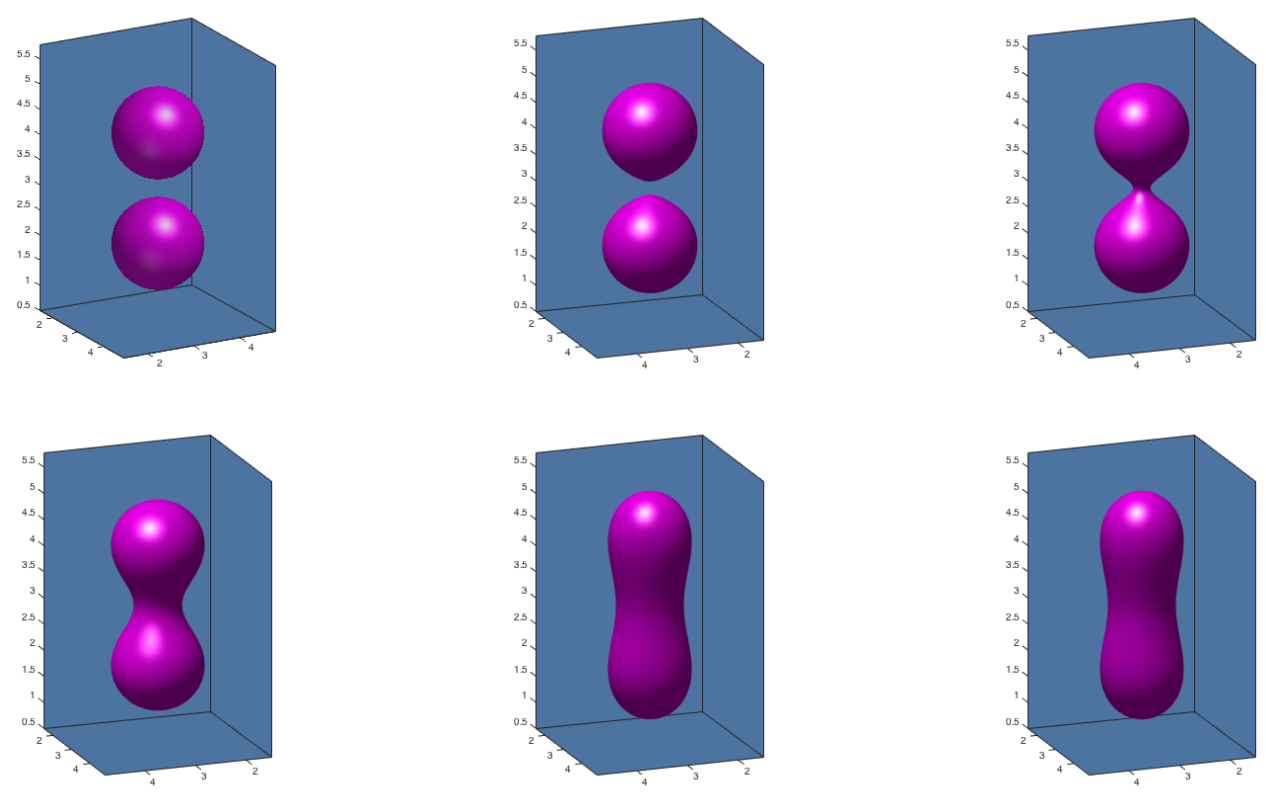

Figure 4.5. The dynamical behaviors of the collision of two 3D close-by spherical vesicles with the surface area and the volume constraints $\left(M_{1}=M_{2}=1000\right)$ using the scheme LS2 with the time step size $\delta t=0.001$. Snapshots of the numerical approximation of $\phi$ are taken at $t=0,0.01,0.02,0.1,2,6$.

and 50000 respectively, where the simulations are done by using the scheme LS2 with the time step $3.125 \times 10^{-5}$ until $t=0.1$. We can clearly observe the monotonic convergence of the simulation results along the increasing of the penalty parameters $M_{1}, M_{2}$.

4.3. Deformation of an ellipsoid. In this example, we simulate the deformation of a 3D ellipsoid with the initial profile as

$$
\phi(x, y, z, 0)=\tanh \left(\frac{1-(x-\pi)^{2} / 4-(y-\pi)^{2}-(z-\pi)^{2}}{2 \epsilon}\right) .
$$

We use the second order scheme LS2 and set the time step as $\delta t=0.001$. For the case without the volume and surface area constraints $\left(M_{1}=M_{2}=0\right)$, snapshots are taken at $t=0,0.16,1.8,3.4$ and 6, shown in Fig. 4.8. The ellipsoid contracts and gradually turns into the spherical shape. When imposing the fixed volume and surface area constraints with the penalty parameters $M_{1}=M_{2}=1000$, the ellipsoid instead transforms into the capsule shape as shown in Fig. 4.9. In Fig. 4.10, we show the time evolution of the energy, the actual volume and the actual surface area for the two cases until $t=6$ (steady states are reached). For the case without the volume and surface area constraints, the energy decreases from 140 to 40 initially in a very small time range (from $t=0$ to around $t=0.01$ ), and then decreases slowly until the steady state. The volume and surface area decrease as well until the steady state. For the case with the fixed volume and surface area constraint, the energy initially decreases sharply, which is similar to the no-constraint case. However, it only takes around $t=0.3$ transition time to reach the steady state. During the transformation process, we observe that the surface area and the 

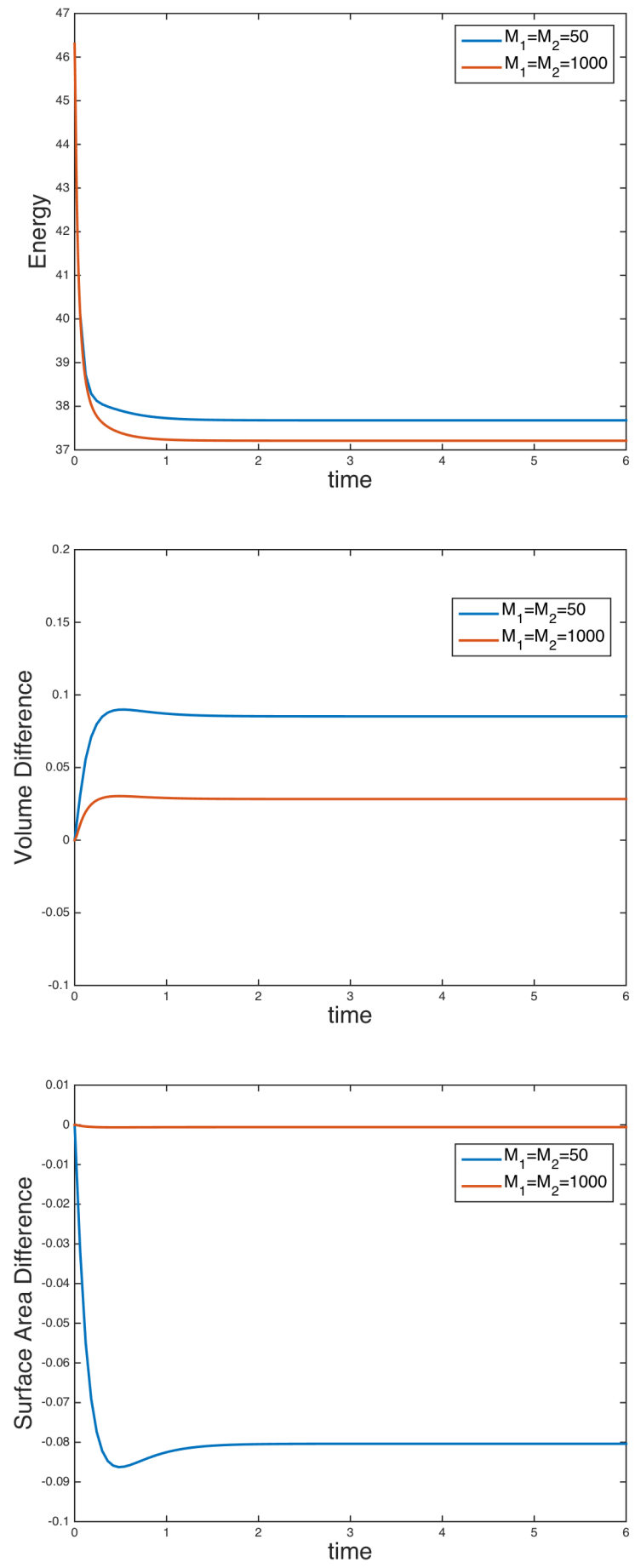

Figure 4.6. Comparison of the time evolution of the free energy, the scaled volume difference $V_{S}(\phi)-\alpha$, and the scaled surface area difference $A_{S}(\phi)-\beta$ for the collision of two $3 \mathrm{D}$ close-by spherical vesicles with the volume and surface area constraints with the penalty parameters $M_{1}=M_{2}=50$ and $M_{1}=M_{2}=1000$ using the scheme LS2 with the time step size $\delta t=0.001$. 

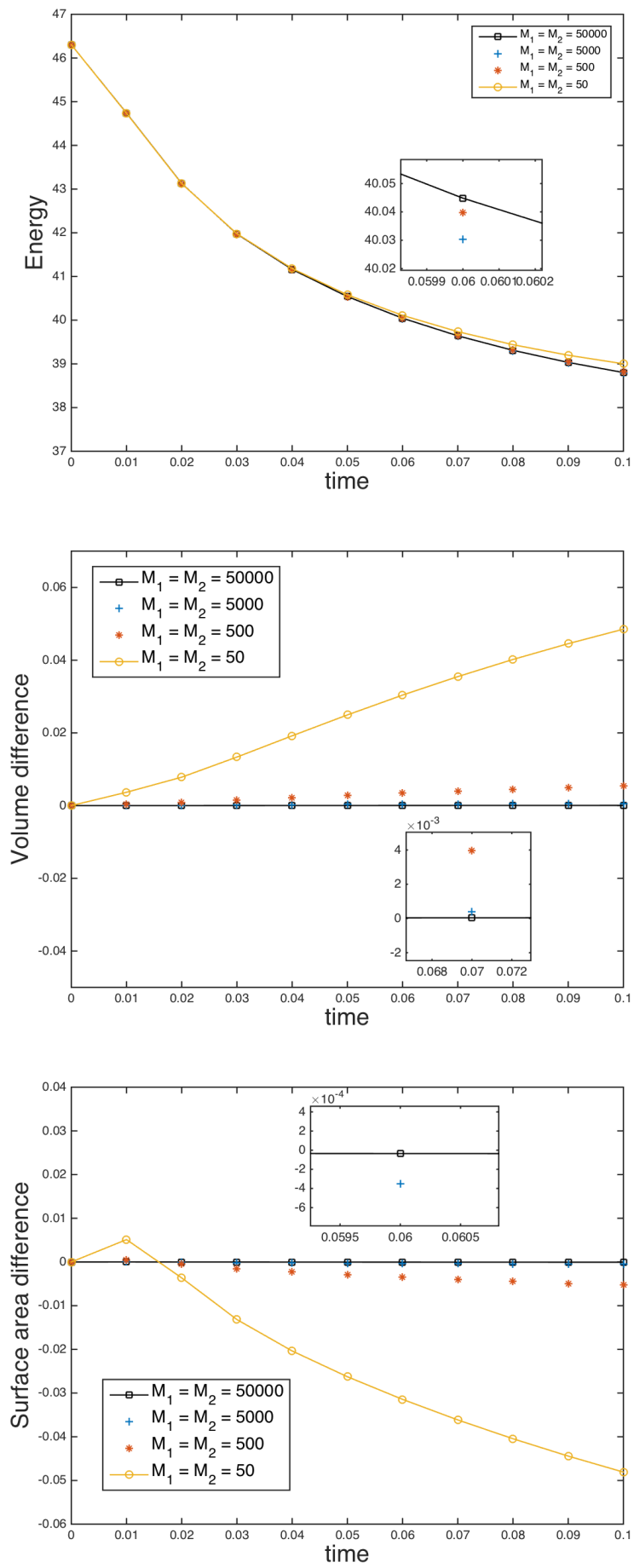

Figure 4.7. Comparison of the time evolution of the free energy, the scaled volume difference $V_{S}(\phi)-\alpha$, and the scaled surface area difference $A_{S}(\phi)-\beta$ for the collision of two $3 \mathrm{D}$ close-by spherical vesicles with the volume and surface area constraints with the penalty parameters $M_{1}=M_{2}=50,500,5000$ and 50000, respectively, using the scheme LS2 with the time step size $\delta t=3.125 \times 10^{-5}$. 

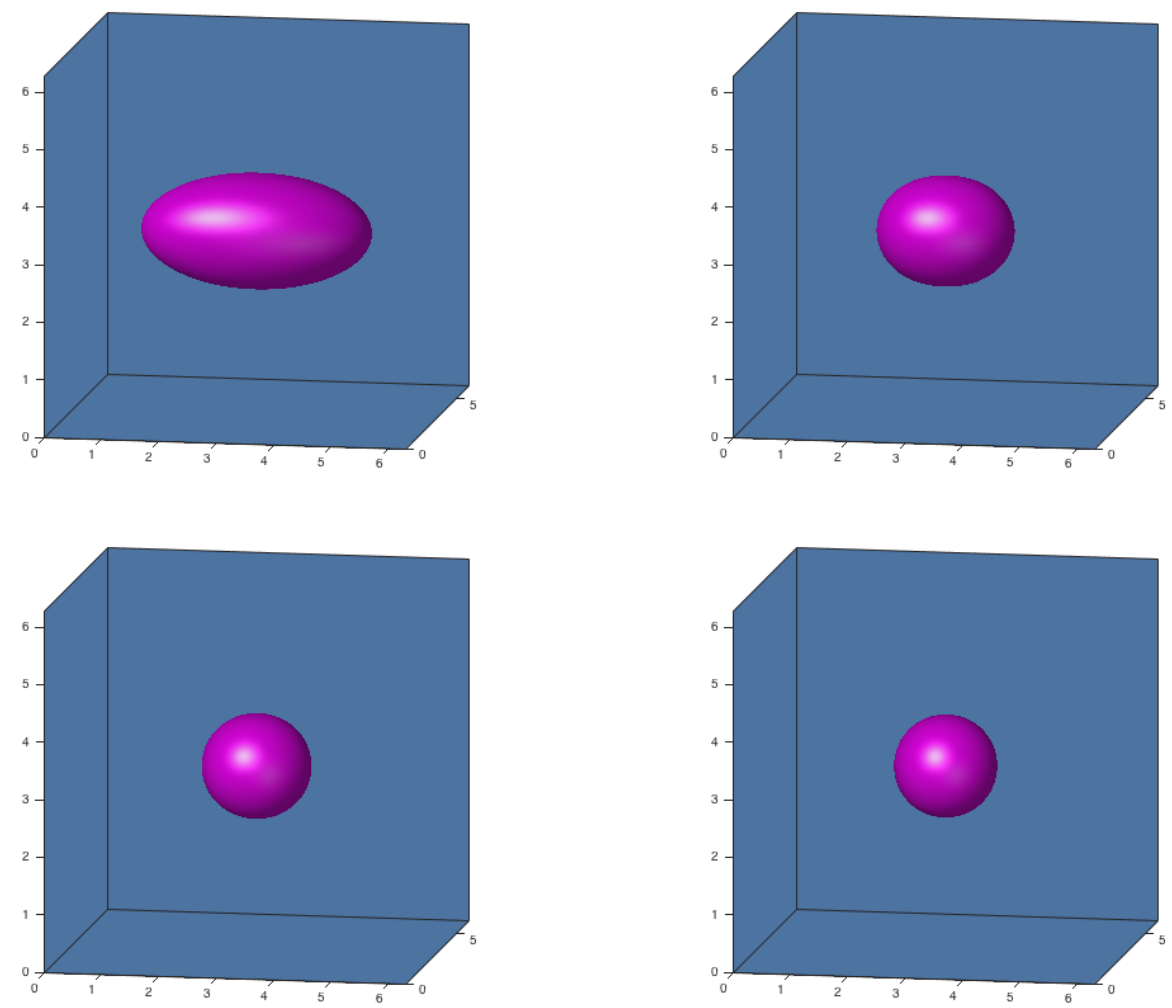

FIGURE 4.8. The dynamical behaviors of an ellipsoid without constraints simulated by the LS2 scheme with the time step size $\delta t=0.001$. Snapshots of the numerical approximation of $\phi$ are taken at $t=0,1.8,3.4$, and 6 .

volume always maintain well at their original values (shown in the last two subfigures in Fig. 4.10).

\section{Concluding Remarks}

In this paper, we have presented two linear, semi-discrete in time, first and second order schemes that are provably unconditionally energy stable for solving the PF-EBE model based on the novel IEQ approach. Both proposed schemes are the first such schemes that are (i) accurate (up to second order in time); (ii) stable (the energy dissipation law holds); and (iii) efficient and easy to implement (only need to solve a linear system with symmetric positive definite operator at each time step). Some of future work includes rigorous convergence and error analysis for fully discretized systems (for instance, by finite element, finite difference or spectral approximations in space) of the PF-EBE model based on the proposed time stepping schemes, and their applications to numerical solution of any related phase field models in biology and materials science. 

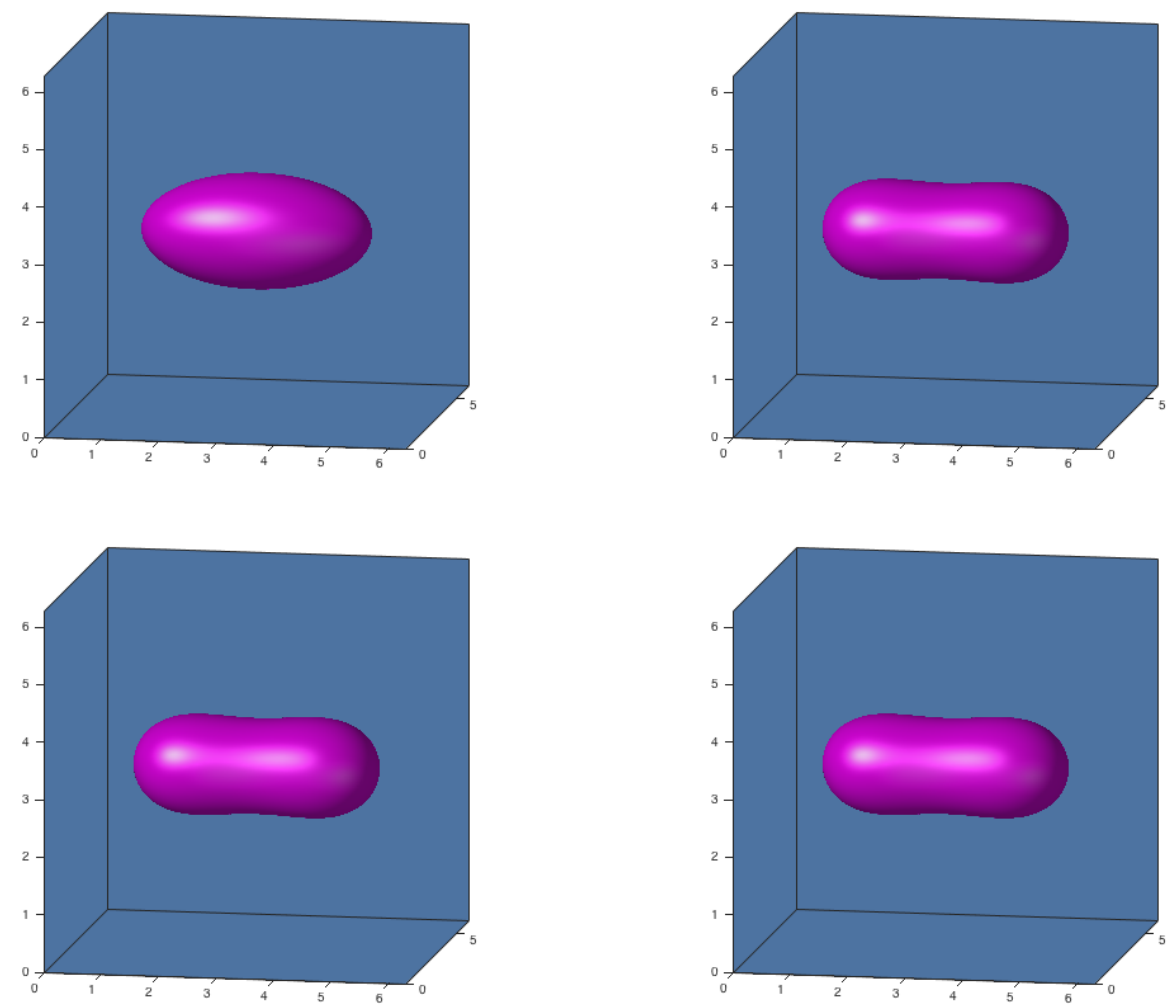

Figure 4.9. The dynamical behaviors of an ellipsoid with the volume and surface area constraints $\left(M_{1}=M_{2}=1000\right)$ simulated by the LS2 scheme with the time step size $\delta t=0.001$. Snapshots of the numerical approximation of $\phi$ are taken at $t=0,1.8,3.4$, and 6 .

\section{ACKNOWLEDGMENTS}

X. Yang's research is partially supported by the U.S. National Science Foundation under grant numbers DMS-1200487 and DMS-1418898, the U.S. Air Force Office of Scientific Research under grant number FA9550-12-1-0178. L. Ju's research is partially supported by the U.S. National Science Foundation under grant number DMS-1521965 and the U.S. Department of Energy under grant number DE-SC0008087-ER6539.

\section{REFERENCES}

[1] Y. Barenholz, D. Gibbes, B. J. Litman, J. Goll, T. E. Thompson, and F. D. Carlson. A simple method for the preparation of homogeneous phospholipid vesicles. Biochemistry, 16:280610, 1977.

[2] S. Batzri and E. D. Korn. Single bilayer liposomes prepared without sonication. Biochim. Biophys. Acta, 298:1015, 1973.

[3] S. J. Biller, F. Schubotz, S. E. Roggensack, A. W. Thompson, R. E. Summons, and S. W. Chisholm. Bacterial vesicles in marine ecosystems. Science, 343:183-186, 2014.

[4] J. W. Cahn and J. E. Hilliard. Free energy of a nonuniform system I. interfacial free energy. J. Chem. Phys., 28:258-267, 1958. 

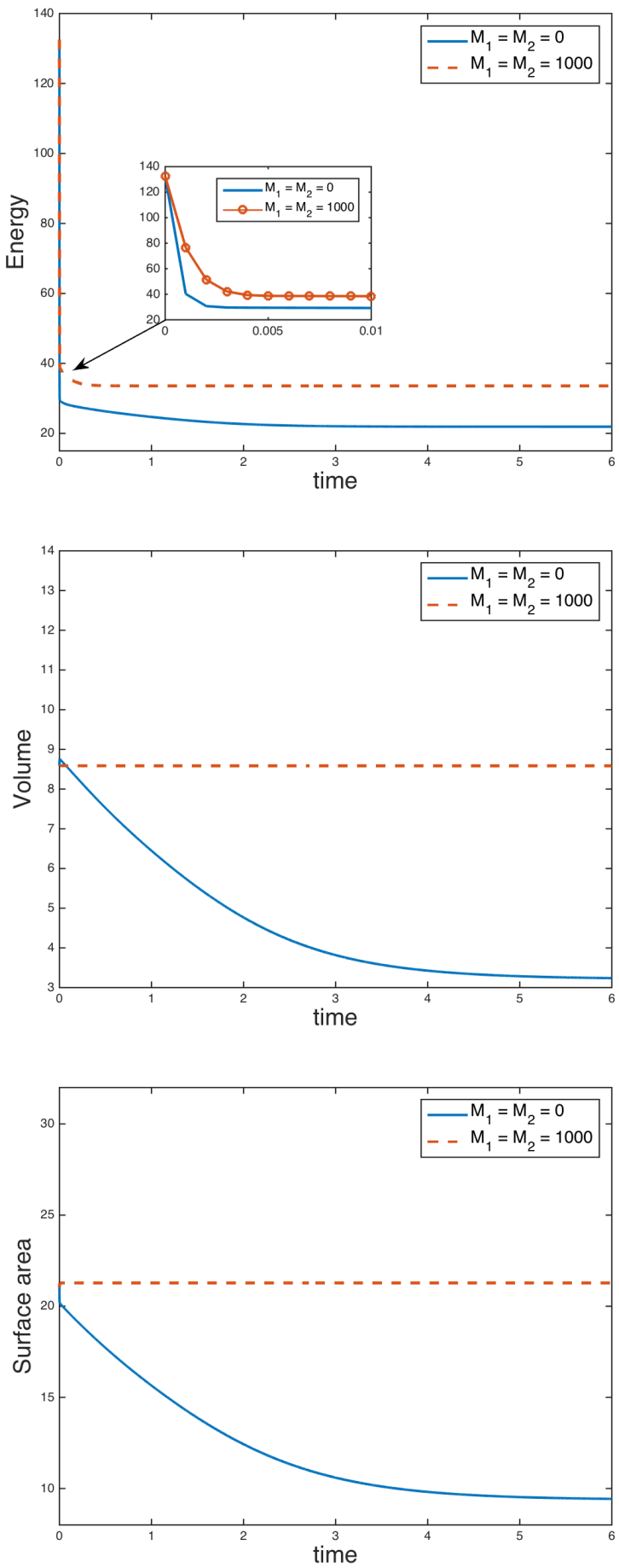

Figure 4.10. Time evolution of the free energy, the actual volume $\frac{1}{2} V_{S}(\phi)$ and the actual surface area $\frac{3}{2 \sqrt{2}} A_{S}(\phi)$ for the example of the deformation of an ellipsoid, with two different penalty parameters where $M_{1}=M_{2}=0$ and 1000 respectively, using the scheme LS2 and the time step size $\delta t=0.001$. 
[5] P. B. Canham. The minimum energy of bending as a possible explanation of the biconcave shape of the human red blood cell. J. Theor. Biol., 26:61-76, 1970.

[6] R. S. Chadwick. Axisymmetric indentation of a thin incompressible elastic layer. SIAM J. Appl. Math., 62:1520-1530, 2002.

[7] L. Q. Chen and Y. Wang. The continuum field approach to modeling microstructural evolution. JOM, 48:13$18,1996$.

[8] R. Chen, G. Ji, X. Yang, and H. Zhang. Decoupled energy stable schemes for fluid vesicle membrane phase field model. J. Comput. Phys., 302:509-523, 2015.

[9] R. Chen, G. Ji, X. Yang, and H. Zhang. Decoupled energy stable schemes for phase-field vesicle membrane model. J. Comput. Phys., 302:509-523, 2015.

[10] Q. Du, M. Li, and C. Liu. Analysis of a phase field navier-stokes vesicle-fluid interaction model. Dis. Conti. Dyn. Sys.-B, 8(3):539-556, 2007.

[11] Q. Du, C. Liu, R. Pyham, and X. Wang. Modeling vesicle deformations in flow fields via energetic variational approaches. prerint, 2006.

[12] Q. Du, C. Liu, R. Ryham, and X. Wang. A phase field formulation of the willmore problem. Nonlinearity, 18:1249-1267, 2005.

[13] Q. Du, C. Liu, and X. Wang. A phase field approach in the numerical study of the elastic bending energy for vesicle membranes. J. Comput. Phys., 198:450-468, 2004.

[14] Q. Du, C. Liu, and X. Wang. Retrieving topological information for phase field models. SIAM J. Appl. Math., 65:1913-1932, 2005.

[15] Q. Du, C. Liu, and X. Wang. Simulating the deformation of vesicle membranes under elastic bending energy in three dimensions. J. Comput. Phys., 212:757-777, 2005.

[16] D. A. Edwards, H. Brenner, and D. T. Wasan. Interfacial transport process and rheology. Butterworths/Heinemann, London, 1991.

[17] A. Evans. Bending resistance and chemically induced moments in membrane bilayers. Bio-phys. J., 14:923931, 1974.

[18] D. J. Eyre. Unconditionally gradient stable time marching the Cahn-Hilliard equation. In Computational and mathematical models of microstructural evolution (San Francisco, CA, 1998), volume 529 of Mater. Res. Soc. Sympos. Proc., pages 39-46. MRS, Warrendale, PA, 1998.

[19] C. Funkhouser, F. Solis, and K. Thorton. Coupled composition-deformation phase-field method for multicomponent lipid membranes. Phys. Rev. E, 76:011912, 2007.

[20] R. Gu, X. Wan, and M. Gunzburger. A two phase field model for tracking vesicle-vesicle adhesion. J. Math. Bio., submitted, 2014.

[21] F. Guillén-González and G. Tierra. On linear schemes for a Cahn-Hilliard diffuse interface model. J. Comput. Phys., 234:140-171, 2013.

[22] D. Han, A. Brylev, X. Yang, and Z. Tan. Numerical analysis of second order, fully discrete energy stable schemes for phase field models of two phase incompressible flows. to appear, J. Sci. Comput., 2016.

[23] W. Helfrich. Elastic properties of lipid bilayers: Theory and possible experiments. Z. Naturforsch. Teil C, 28:693-703, 1973.

[24] A. Ratz J. Lowengrub and A. Voigt. Phase-field modeling of the dynamics of multicomponent vesicles: Spinodal decomposition, coarsening, budding, and fission. Phys. Rev. E., 79:031926, 2009.

[25] J. Kim. Phase-field models for multi-component fluid flows. Comm. Comput. Phys, 12(3):613-661, 2012.

[26] V. V. Krotov and A.I. Rusanov. Physicochemical hydrodynamics of capillary systems. Imperial College Press, London, 1999.

[27] M. J. Kuehn and N. C. Kesty. Bacterial outer membrane vesicles and the host-pathogen interaction. Genes \& Development, 19:26452655, 2005.

[28] R. Lipowsky. The morphology of lipid menbranes current opinion in structural biology. Comm. Pure Appl. Math., XLV, 58:501-537, 1995.

[29] C. Liu and J. Shen. A phase field model for the mixture of two incompressible fluids and its approximation by a Fourier-spectral method. Physica D, 179(3-4):211-228, 2003.

[30] J. S. Lowengrub, A. Ratz, and A. Voigt. Phase field modeling of the dynamics of multicomponent vesicles spinodal decomposition coarsening budding and fission. Physical Review E, 79(3), 2009. 
[31] C. Miehe, M. Hofacker, and F. Welschinger. A phase field model for rate-independent crack propagation: Robust algorithmic implementation based on operator splits. Computer Methods in Applied Mechanics and Engineering, 199:2765-2778, 2010.

[32] P. G. H. Nayanajith, S. C. Saha, and Y. T. Gu. Deformation properties of single red blood cell in a stenosed microchannel. APCOM ISCM, 58:94-144, 11-14th December, (2013), Singapore.

[33] R. F. Probstein. Physicochemical hydrodynamics: An introduction. Wiley, New York, 1994.

[34] L. Rayleigh. On the theory of surface forces II. Phil. Mag., 33:209, 1892.

[35] J. Shen and X. Yang. Numerical approximations of allen-cahn and cahn-hilliard equations. DCDS, Series A, 28:1169-1691, 2010.

[36] J. Shen and X. Yang. A phase field model and its numerical approximation for two phase incompressible flows with different densities and viscosities. SIAM Journal of Scientific Computing, 32(3):1159-1179, 2010.

[37] V. K. Sidhu, F.. J. Vorhlter, K. Niehaus, and S. A. Watt. Analysis of outer membrane vesicle associated proteins isolated from the plant pathogenic bacterium xanthomonas campestris pv. campestris. BMC Microbiol., $8: 87,2008$.

[38] D. Siegel and M. Kozlov. The gaussian curvature elastic modulus of $\mathrm{n}$-monomethylated dioleoylphosphatidylethanolamine: Relevance to membrane fusion and lipid phase behavior. Biophys J., 87:366-374, 2004.

[39] R. Spatschek, E. Brener, and A. Karma. A phase field model for rate-independent crack propagation: Robust algorithmic implementation based on operator splits. Philosophical Magazine, 91:75-95, 2010.

[40] J. van der Waals. The thermodynamic theory of capillarity under the hypothesis of a continuous density variation. J. Stat. Phys., 20:197-244, 1893.

[41] A. E. Walsby. Gas vesicles. Microbiological reviews, 58:94-144, 1994.

[42] Q. Wang, S. Sircar, and H. Zhou. Steady state solutions of the smoluchowski equation for nematic polymers under imposed fields. Communications in Mathematical Sciences, 4:605-620, 2005.

[43] X. Wang. Astmptotic analysis of phase field formulations of bending elasticity models. SIAM J. Math. Anal., 39:1367-1401, 2008.

[44] X. Wang and Q. Du. Modelling and simulations of multi-component lipid membranes and open membranes via diffusive interface approaches. J. Math. Bio., 56:347-371, 2008.

[45] X. Wang, L. Ju, and Q. Du. Efficient and stable exponential time differencing runge-kutta methods for phase field elastic bending energy models. J. Comput. Phys., 316:21-38, 2016.

[46] C. Xu and T. Tang. Stability analysis of large time-stepping methods for epitaxial growth models. Liq. Cryst., 44:1759-1779, 2006.

[47] X. Yang and D. Han. Linearly first- and second-order, unconditionally energy stable schemes for the phase field crystal equation. to appear, J. Comput. Phys., 2016.

[48] J. Zhang, S. Das, and Q. Du. A phase field model for vesicle-substrate adhesion,. J. Comput. Phys., 228:78377849, 2009. 